\title{
Period of the Slichter mode of Mercury and its possible observation
}

\author{
A. Coyette ${ }^{1,2}$, T. Van Hoolst ${ }^{1}$, and V. Dehant ${ }^{1,2}$
}

\author{
${ }^{1}$ Royal Observatory of Belgium, Ringlaan 3, 1180 Brussels, Belgium \\ e-mail: alexis . coyette@observatoire.be \\ 2 Université catholique de Louvain, Earth and Life Institute, Georges Lemaitre Centre for Earth and Climate Research, Belgium
}

Received 26 January 2012 / Accepted 17 April 2012

\section{ABSTRACT}

\begin{abstract}
Aims. We study the period of the Slichter mode (vibrational mode of the inner core of a planet) of Mercury in relation to its interior structure and assess the possibility to observe this mode with the probes MESSENGER and BepiColombo.

Methods. The methodology of Grinfeld \& Wisdom (2005, Phys. Earth Planet. Int., 151, 77) for the determination of the period of the polar Slichter modes of a planetary interior consisting of three homogeneous layers is generalized to models with an arbitrary but finite number of layers. Slichter modes periods are calculated for a large set of interior structure models of Mercury. We study the possible excitation of Slichter modes by a colllision with a meteoroid and estimate the minimal size of the meteoroid that could lead to a detection of these modes by BepiColombo.

Results. The Slichter mode period obtained is on the order of several hours. Observation of the Slichter mode of Mercury allows constraining the inner core. An impact by a meteoroid with a radius of at least $100 \mathrm{~m}$ could excite the Slichter mode to a level observable by BepiColombo (assuming that the Slichter mode is the only excited mode), but since the estimated damping time of the Slichter mode is well below the average time between impacts of at least that size such an impact must have occured recently (less than 0.5 My ago).
\end{abstract}

Key words. planets and satellites: individual: Mercury - planets and satellites: interiors - planets and satellites: physical evolution planetary systems

\section{Introduction}

The Slichter modes of a terrestrial planet are vibrational modes of the inner core of the planet corresponding to translational oscillations of the inner core within the outer core. These modes are referred to as the Slichter modes as they were theoretically introduced, but not observed, by Slichter (1961) after the earthquake in Chile, in 1960. With the development of superconducting gravimeters, the quest for the experimental discovery of the Slichter modes of the Earth was boosted. The Slichter modes of the Earth are yet to be detected (e.g. Jensen et al. 1995; Hinderer et al. 1995; Kroner et al. 2004; Sun et al. 2006; Rosat et al. 2007) even though some scientists already claimed their detection (e.g. Smylie 1992; Courtier et al. 2000; Xu et al. 2010). The search for the detection of the Slichter modes of the Earth still continues.

The high mean density of Mercury compared to the other terrestrial planets is thought to imply that the core of Mercury is relatively much larger than the core of the other terrestrial planets of the solar system (about $60 \%$ or more of the mass of Mercury, Rivoldini et al. 2009, compared to 32\% for the Earth). Many factors also seem to indicate the presence of an inner core. Observations of periodic changes in Mercury's spin rate have shown that at least the outer part of Mercury's core is liquid (Margot et al. 2007). The global magnetic field (observed by Mariner 10 in 1974 and confirmed by MESSENGER, Anderson et al. 2008), if due to a hydrodynamo, can be considered as evidence of the presence of a growing inner core, although other hypotheses for the origin of the magnetic field have been studied (as crustal magnetization (e.g. Aharonson et al. 2004) or thermoelectric dynamo (e.g. Giampieri \& Balogh 2002). An inner core is also consistent with thermal evolution models of Mercury although they cannot rule out a fully liquid core (Hauck II et al. 2004; Breuer et al. 2007), and with the lobate scarps on the surface of Mercury (e.g. Melosh \& McKinnon 1988; Watters et al. 2004, 2009), which are thought to be created by the contraction of the planet and may partly be caused by the formation of a solid inner core.

The motivation for this study is that, thanks to its possibly larger inner core (due to its larger core), the Slichter modes of Mercury could be easier to detect than the Slichter modes of the Earth. Therefore, we study the possibility of observation by probes like MESSENGER - now in orbit about Mercury since March 18, 2011 - or BepiColombo - probably in orbit about Mercury in 2020.

The first analytical study to determine the period of the Slichter modes of the Earth was performed by Busse (1974). Busse considered an Earth divided into a static rigid mantle, a homogeneous fluid outer core and a rigid inner core, and assumed that no mantle motion is associated with the Slichter modes. More recently, Grinfeld \& Wisdom (2005) developed a method for a model of the Earth consisting of three homogeneous layers that takes into account the motion of the mantle.

The internal structure of Mercury being still unknown, we first develop a set of three-layer models of Mercury and use them in order to calculate the Slichter mode period of Mercury (Sect. 2). We also extend, in Sect. 3, the model of Grinfeld \& Wisdom (2005) to calculate the Slichter mode for models with stratified layers. Finally, we assess the possibilities for orbiting spacecraft to observe the Slichter mode of Mercury using a simple model of excitation of the Slichter mode due to collision with a meteoroid (Sect. 4). 


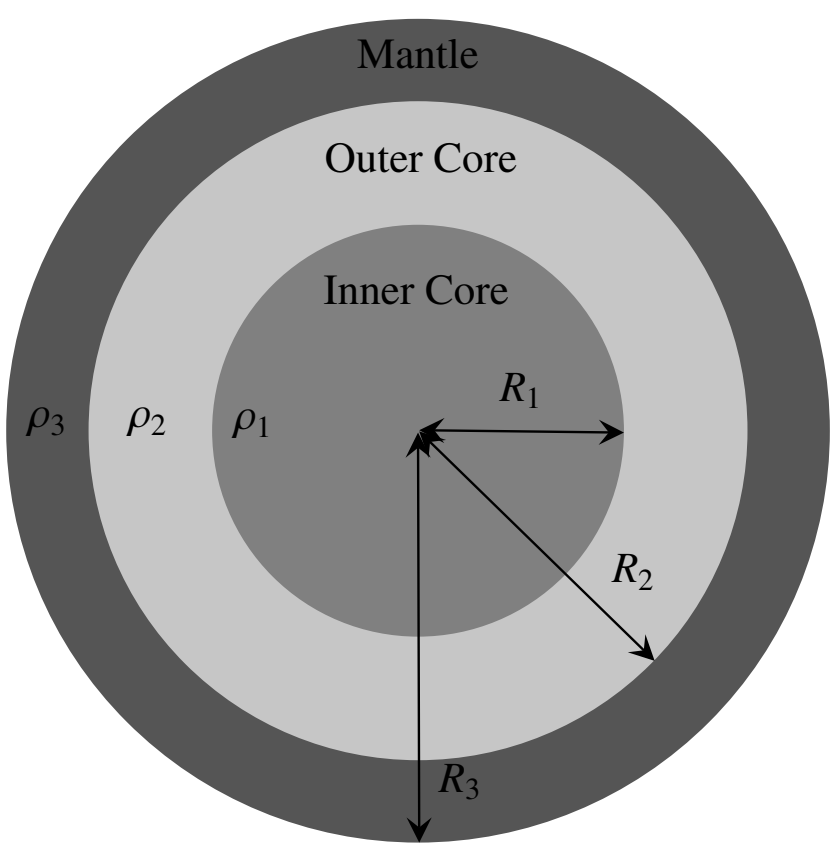

Fig. 1. Interior structure of Mercury consisting of a solid inner core, a liquid outer core and a solid mantle.

\section{Models of Mercury}

In order to obtain the period of the Slichter modes of Mercury, we consider and generalize the methodology developed by Grinfeld \& Wisdom (2005) for a three-layer model of Mercury. We therefore first consider models of the interior structure of Mercury in which the mantle, the outer core, and the inner core are of constant density. We divide Mercury into three homogeneous layers of density $\rho_{k}$, radius $R_{k}$ and mass $M_{k}$, as represented in Fig. 1. The subscript $k$ takes values 1,2 and 3 for the inner core, the outer core and the mantle, respectively. The goal of this section is to derive basic three-layer models of Mercury with varying inner core radius and core sulfur concentration in order to be able to assess the impact of these parameters on the Slichter mode. To develop these models, we will use the methodology developed in Van Hoolst \& Jacobs (2003).

As many nickel-iron meteorites contain sulfur under the form FeS, sulfur can be considered as a major candidate for light-elements in planetary cores of terrestrial planets (Rivoldini et al. 2009). Moreover, sulfur has a high solubility in molten iron and can therefore be more easily incorporated into planetary cores during the process of core formation. We will here consider that the core of Mercury is only composed of iron and sulfur. Other light (oxygen, carbon...) or heavy (nickel) elements are neglected. We first consider interior structure models of Mercury with an entirely liquid core and a range of sulfur concentration of the core $x_{\mathrm{S}}$ between $0.1 \mathrm{wt} \%$ (almost pure iron core) and $14 \mathrm{wt} \%$. This last value is chosen to include models with a light element concentration as high as that of Mars (Dreibus \& Wänke 1985). The mass concentration of $\mathrm{FeS}, x_{\mathrm{FeS}}$, corresponding to these sulfur concentrations $x_{S}$ can be calculated using

$x_{\mathrm{FeS}}=x_{\mathrm{S}} \frac{M_{\mathrm{FeS}}}{M_{\mathrm{S}}}$

where $M_{\mathrm{X}}$ stands for the molar mass of the component X. For a given core concentration, we first calculate an interior structure model with an entirely liquid core and then consider increasingly larger inner cores until the core is entirely solid. When the core
Table 1. Thermoelastic properties of Fe and FeS (solid and liquid).

\begin{tabular}{lcccc}
\hline \hline Thermoelastic & \multicolumn{2}{c}{ Solid } & \multicolumn{2}{c}{ Liquid } \\
properties & $\gamma \mathrm{Fe}^{a}$ & $\mathrm{FeS} \mathrm{IV}$ & $\mathrm{Fe}^{c}$ & $\mathrm{FeS}$ \\
\hline$T_{\text {ref }}(\mathrm{K})$ & 1573 & 600 & 1811 & $1800^{d}$ \\
$V\left(\mathrm{~cm}^{3} / \mathrm{mole}\right)$ & 7.53 & 17.16 & 7.96 & $24.74^{d}$ \\
$\alpha\left(10^{-5} \mathrm{~K}^{-1}\right)$ & 7.7 & $7.16(51)$ & 9.20 & $16.28^{d}$ \\
& & $+6.08(1.31) \mathrm{T}$ & & \\
$K_{T}(\mathrm{GPa})$ & 103 & $62.5(9)$ & 85 & $20(2)^{e}$ \\
$K_{T}^{\prime}$ & 5 & 4 & 5.8 & $8^{e}$ \\
$\mathrm{~d} K_{T} / \mathrm{d} T(\mathrm{GPa} / \mathrm{K})$ & -0.020 & $-0.0208(28)$ & -0.031 & -0.0208 \\
\hline
\end{tabular}

Notes. The accuracy of experimental data are indicated in brackets. The value of $\mathrm{d} K / \mathrm{d} T$ for liquid $\mathrm{FeS}$ is taken equal to the value for solid FeSIV.

References. (a) Ahrens et al. (2002); (b) Urakawa et al. (2004); (c) Anderson \& Ahrens (1994); ${ }^{(d)}$ Kaiura \& Toguri (1979); ${ }^{(e)}$ Nishida et al. (2008).

is entirely liquid, the density of the mantle, $\rho_{3}$, is assumed to be $3500 \mathrm{~kg} / \mathrm{m}^{3}$ (Van Hoolst \& Jacobs 2003; Rivoldini et al. 2009).

Since sulfur does not dissolve well in solid Fe at Mercury core pressures ( $\mathrm{Li}$ et al. 2001), we consider that pure iron precipitates to form the inner core. As a result, the inner core is assumed to consist of pure $\gamma$-Fe and the outer of a Fe-FeS solution. The pressure inside Mercury's core varies from about $3 \mathrm{GPa}$ at the core-mantle boundary to $44 \mathrm{GPa}$ at the center of Mercury (Rivoldini et al. 2009). We assume here a constant density of $20 \mathrm{GPa}$ in the whole core. The temperature inside Mercury's core is taken equal to $2200 \mathrm{~K}$. The densities of the different core layers are calculated from the density of iron and iron-sulfur at reference conditions (Table 1) by correcting separately for temperature and pressure. In particular, for the liquid outer core, we will not assume as Van Hoolst \& Jacobs (2003) that the density of liquid $\mathrm{FeS}$ is $3.5 \%$ lower than solid FeS, but we will use new observational data for liquid FeS (Nishida et al. 2008).

First we correct for temperature. For each element, such as $\gamma$-Fe in the inner core, the dependence of density on temperature is given in terms of the coefficient of thermal expansivity

$\alpha(T)=\left.\frac{\partial \ln V(T)}{\partial T}\right|_{P}$

as

$\rho_{0}(T)=\rho_{\text {ref }} \exp \left[-\int_{T_{\text {ref }}}^{T} \mathrm{~d} T^{\prime} \alpha\left(T^{\prime}\right)\right]$

where the subscript 0 indicates that the density is calculated at the reference pressure and $\rho_{\text {ref }}$ is the density at reference pressure and temperature (Table 1). For the liquid solution of $\mathrm{Fe}$ and $\mathrm{FeS}$, we assume that it can be described by an ideal solution model, i.e. the molar volume of the solution is the sum of the molar volumes of the components weighted by their molar fraction. The molar volume of the composition of the core at reference pressure and local temperature $T$ can then be expressed as

$V_{0}\left(\chi_{\mathrm{FeS}}, T\right)=\chi_{\mathrm{FeS}} V_{\mathrm{FeS}}(T)+\left(1-\chi_{\mathrm{FeS}}\right) V_{\mathrm{Fe}}(T)$

with $\chi_{\mathrm{FeS}}$ the molar fraction of $\mathrm{FeS}$ and $V_{\mathrm{Fe}(\mathrm{S})}$ the molar volume of $\mathrm{Fe}(\mathrm{S})$ at temperature $T$ (calculated using Eq. (3)). In a solution of $\mathrm{A}$ and $\mathrm{B}$, the relation between mass fraction $x_{\mathrm{A}}$ and molar fraction $\chi_{\mathrm{A}}$ is given by

$\chi_{\mathrm{A}}=\left[\left(\frac{1}{x_{\mathrm{A}}}-1\right) \frac{M_{\mathrm{A}}}{M_{\mathrm{B}}}+1\right]^{-1}$. 
The density of a solution of Fe and FeS is then calculated from

$\rho_{0}=\frac{\chi_{\mathrm{FeS}} M_{\mathrm{FeS}}+\left(1-\chi_{\mathrm{FeS}}\right) M_{\mathrm{Fe}}}{V_{0}}$

We next correct densities for pressure, using the third-order Birch-Murnaghan isothermal equation of state

$$
\begin{aligned}
P=\frac{3}{2} K_{T, 0} & {\left[\left(\frac{\rho}{\rho_{0}}\right)^{7 / 3}-\left(\frac{\rho}{\rho_{0}}\right)^{5 / 3}\right] } \\
& \times\left(1+\frac{3}{4}\left(K_{T, 0}^{\prime}-4\right)\left[\left(\frac{\rho}{\rho_{0}}\right)^{2 / 3}-1\right]\right) .
\end{aligned}
$$

Here $K_{T}$ is the bulk modulus given, at reference temperature, by

$$
K_{T, \text { ref }}=-\left.\frac{\partial P}{\partial \ln V}\right|_{T},
$$

while $K_{T}^{\prime}$ is its derivative with respect to pressure. At temperature $T$, the bulk modulus is given by

$$
K_{T, 0}(T)=K_{T, \text { ref }}+\left.\left(\frac{\mathrm{d} K_{T}}{\mathrm{~d} T}\right)\right|_{P}\left(T-T_{\text {ref }}\right) .
$$

The bulk modulus of a solution of $\mathrm{Fe}$ and $\mathrm{FeS}$, and its derivative with respect to pressure are given by (Rivoldini et al. 2011)

$$
K_{T, 0}=-V_{0}\left(\frac{\chi_{\mathrm{FeS}} V_{\mathrm{FeS}}}{K_{T, 0, \mathrm{FeS}}}+\frac{\left(1-\chi_{\mathrm{FeS}}\right) V_{\mathrm{Fe}}}{K_{T, 0, \mathrm{Fe}}}\right)^{-1}
$$

and

$$
\begin{aligned}
K_{T, 0}^{\prime}=-1 & +\frac{K_{T, 0}^{2}}{V_{0}}\left[\frac{\chi_{\mathrm{FeS}} V_{\mathrm{FeS}}}{K_{T, 0, \mathrm{FeS}}^{2}}\left(1+K_{T, \mathrm{FeS}}^{\prime}\right)\right. \\
+ & \left.\frac{\left(1-\chi_{\mathrm{Fe}}\right) V_{\mathrm{Fe}}}{K_{T, 0, \mathrm{Fe}}^{2}}\left(1+K_{T, \mathrm{Fe}}^{\prime}\right)\right] .
\end{aligned}
$$

As before, subscripts 0 indicate the value at local $T$ and reference pressure.

Given a concentration of FeS in the core of Mercury we can calculate the mean density of the core as the density of the solution at a pressure of $20 \mathrm{GPa}$ and a temperature of $2200 \mathrm{~K}$. For an entirely liquid core, we have only two layers, the mantle and the core in which we consider concentrations of sulfur of $0.1,2$, $4,6,8,10,12$ and $14 \mathrm{wt} \%$. The radius of the core $R_{2}$ is given by the total mass of Mercury

$M=\frac{4 \pi}{3}\left[\rho_{2} R_{2}^{3}+\rho_{3}\left(R_{3}^{3}-R_{2}^{3}\right)\right]$

as

$R_{2}=\left(\frac{3 M / 4 \pi-\rho_{3} R_{3}^{3}}{\rho_{2}-\rho_{3}}\right)^{1 / 3}$,

where $R_{3}$, the radius of Mercury, is $2439 \mathrm{~km}$ (Oberst et al. 2011; Perry et al. 2011).

The cooling of Mercury is at the origin of the inner core formation. When the liquid of the core with sulfur concentration $\chi_{\mathrm{S}}$ cools and locally reaches the liquidus, liquid core material solidifies. Since only iron is considered to solidify, the formation of the inner core causes a release of light elements at the inner core boundary and thus an increase of the sulfur concentration of the outer core. Assuming that there is no mass exchange between the core and the mantle (i.e. the mass of the core is constant), the outer core FeS concentration is given by (Van Hoolst \& Jacobs 2003)

$x_{\mathrm{FeS}}(t)=\frac{x_{\mathrm{FeS}}(0)}{1-\left(M_{1}(t) / M_{\mathrm{c}}\right)}$,

where the increasing mass of the inner core is

$M_{1}(t)=\frac{4 \pi}{3} \rho_{1} R_{1}^{3}(t)$

and $M_{\mathrm{c}}=M_{1}(t)+M_{2}(t)$. The radius of the outer core is obtained from the total mass of the core

$R_{2}=\left(\frac{3 M_{\mathrm{c}} / 4 \pi-\left(\rho_{1}-\rho_{2}\right) R_{1}^{3}}{\rho_{2}}\right)^{1 / 3}$,

while the density of the mantle is given by the total mass of the planet

$\rho_{3}=\frac{3 M / 4 \pi-\rho_{2}\left(R_{2}^{3}-R_{1}^{3}\right)-\rho_{1} R_{1}^{3}}{R_{3}^{3}-R_{2}^{3}}$.

The phase diagram of the Fe-S solution is characterized by a sharp minimum in the liquidus curve. Experimental studies at a pressure of $21 \mathrm{GPa}$ ( $\mathrm{Li} \&$ Fei 2005) show that this point is reached when the sulfur concentration of the outer core is approximately $16 \mathrm{wt} \%$. When the eutectic point is reached, the sulfur of the outer core precipitates along with iron. As iron-sulfur is lighter than iron, the inner core can be divided into two parts: a central iron part (with radius $R_{1}$ and density $\rho_{1}$ ) and an external part with eutectic composition (radius $R_{1}^{\text {eut }}$ and density $\rho_{1}^{\text {eut }}$ ), as represented in Fig. 2. From the eutectic point on, compositional buoyancy in the outer core stops as there is no more sulfur released during the inner core formation. The presence of a global magnetic field seems then to indicate that the inner core of Mercury hasn't yet reached the eutectic point. Moreover, due to the low melting temperature of the eutectic point (between about $1200 \mathrm{~K}$ and $1400 \mathrm{~K}$, Rivoldini et al. 2009), it seems unrealistic that the inner core has already reached the eutectic point. Nevertheless, we here consider also models in which the outer core has reached the eutectic point. For a new solidified layer, the inner core is then assumed to consist of solid $\gamma$-Fe and solid iron-sulfur FeS IV (Ono et al. 2008). The mass of the inner core at the eutectic point can be determined by using Eq. (14)

$M_{1}^{\text {eut }}=M_{\mathrm{c}}\left(1-\frac{x_{\mathrm{FeS}}(0)}{x_{\mathrm{FeS}}^{\mathrm{eut}}}\right)$.

From this equation, we obtain the radius $R_{1}$ of the inner core at the eutectic. Again, the radius and densities of the outer core and of the mantle are calculated using Eqs. (16) and (17).

\section{Slichter modes}

We describe the Slichter mode with the method developed by Grinfeld \& Wisdom (2005) which takes into account the motion of the mantle associated with the Slichter modes. However, the method was applied to models of the interior structure consisting of three homogeneous layers. Since Mercury's interior is better modeled by four layers when the eutectic composition is reached in the outer core, we extend the method to more than three layers. We develop an extension to an arbitrary number $N$ of layers in order to be able to apply it to more general models with stratified layers, e.g. developed by Rivoldini et al. (2009). 


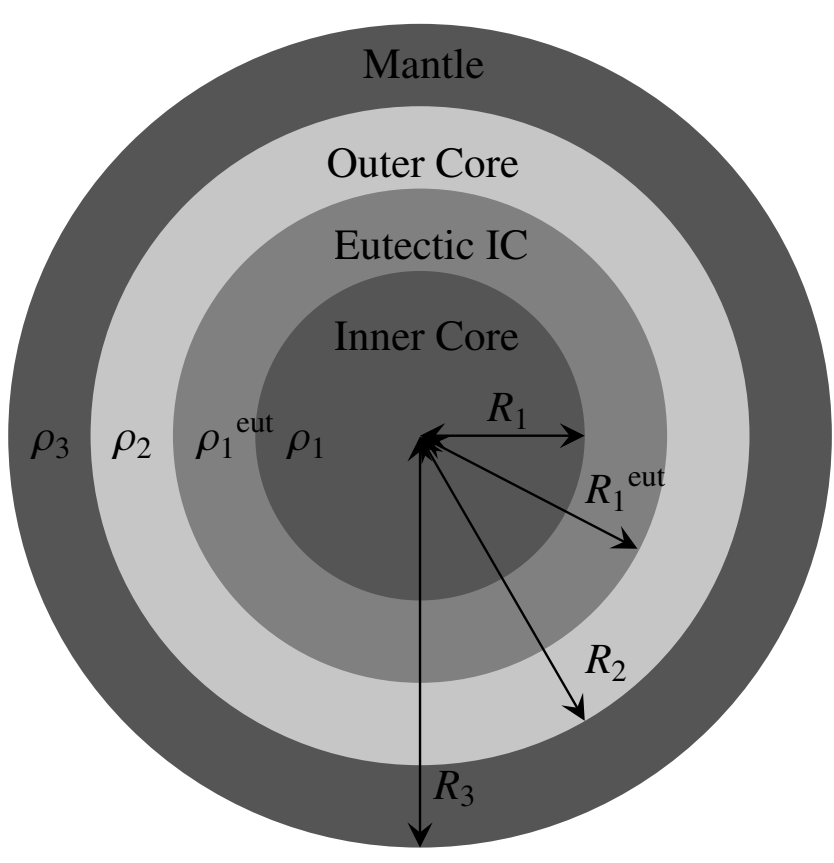

Fig. 2. Interior structure of Mercury once the eutectic point is reached. The inner core is divided into two parts: a central iron part and an external part with eutectic composition.

We consider an inner core divided into ic layers and a mantle divided into $m$ layers. In order to simplify the calculations, we keep only one layer for the outer core so that we have $N=\mathrm{i} c+$ $m+1$ layers in total. An extension to several layers in the outer core is possible but not needed since, as will be shown is Sect. 4, observation of the Slichter mode is only expected for sufficiently large inner cores for which a constant density in the outer core is a good approximation.

We assume that the planet is not rotating. The rotation of the planet leads to a splitting of the Slichter mode into a triplet (Busse 1974). However, due to the slow rotation of Mercury (in 58.6 terrestrial days), the splitting of the Slichter mode can be neglected to a very good approximation (the splitting will be on the order of a few seconds).

Following Grinfeld \& Wisdom (2005), we determine the frequency of the Slicher mode from the equation of conservation of momentum of the planet and from the Newton's second law for the inner core.

\subsection{Equation of conservation of momentum}

We consider a spherically symmetric, static Mercury as the equilibrium state. The spherical symmetry allows us to consider an inner core velocity in only one direction. For simplicity, we choose the $\hat{z}$ direction. The equation of conservation of momentum in the $\hat{z}$ direction is

$\sum_{j=1}^{N} M_{j} v_{j}^{z}=0$

with $v_{j}^{z}=\mathrm{i} \omega \mathrm{e}^{\mathrm{i} \omega t} R_{j} A_{j}^{z}$ where $\omega$ is the frequency of the Slichter mode and $A_{j}^{z}$ is the normalized oscillation amplitude of the $j$-layer along $\hat{z}$.

The normal velocity of an interface $k$ between different layers is expressed in terms of harmonic series

$C_{k}(\theta, \phi)=\mathrm{i} \omega \mathrm{e}^{\mathrm{i} \omega t} R_{k} \sum_{l, m} C_{k}^{l m} Y_{l m}(\theta, \phi)$, where $R_{k}$ is the radius of the $k$ th layer, $\theta$ is the colatitude and $\phi$ the longitude. We use here spherical harmonics $Y_{l m}(\theta, \phi)$ normalized to unity on the unit sphere.

The velocity of an interface can be related to the velocity of adjacent layer. For example, if the $k$ th layer is moving with a velocity $\boldsymbol{v}_{k}=\mathrm{i} \omega \mathrm{e}^{\mathrm{i} \omega t} R_{k} A_{k}^{z} \hat{z}$, the resulting normal velocity $C_{k}$ at the interface is

$$
\begin{aligned}
C_{k} & =\boldsymbol{v}_{k} \cdot N \\
& =\mathrm{i} \omega R_{k} \mathrm{e}^{\mathrm{i} \omega t} \sqrt{\frac{2 \pi}{3}} \sqrt{2} A_{k}^{z} Y_{1,0}
\end{aligned}
$$

so that we have $C_{k}^{10}=\sqrt{4 \pi / 3} A_{k}^{z}$. From comparison with Eq. (20), we obtain the amplitude of the interface velocity $C_{k}^{10}=\sqrt{4 \pi / 3} A_{k}^{z}$ as a function of the amplitude of the inner core oscillation $A_{k}^{z}$. Equation (21) also shows that for an oscillation along the $\hat{z}$ axis, the interface velocity can be fully represented using only harmonics $l=1$ and $m=0$.

For practical purposes, we divide Mercury into $N$ spheres of radius $R_{1}, \ldots$ and $R_{N}$, density $[\rho]_{1}=\rho_{1}-\rho_{2}, \ldots,[\rho]_{N-1}=$ $\rho_{N-1}-\rho_{N}$ and $[\rho]_{N}=\rho_{N}$. The equation of conservation of momentum then becomes

$$
\left(\sum_{j=1}^{i c}[\rho]_{j} R_{j}^{3}\right) R_{i c} C_{i c}^{10}+\left(\sum_{j=i c+1}^{N}[\rho]_{j} R_{j}^{3}\right) R_{N} C_{N}^{10}=0
$$

where subscript ic is used for the inner core and subscript $N$ for the mantle of Mercury. We assume here that the inner core and mantle behave rigidly, which implies that the normal velocities at the surface of the different layers of the inner core and mantle are equal, i.e. $R_{j} A_{j}^{z} \equiv R_{i c} A_{i c}^{z}$ for $j \in\{1, \ldots, i c\}$ and $R_{j} A_{j}^{z} \equiv R_{N} A_{N}^{z}$ for $j \in\{i c+1, \ldots, N\}$. Note that, unlike Grinfeld \& Wisdom (2005), we will not use nondimensional expressions in order to be able to verify the dimension of our different equations.

The ratio between the amplitude of the normal velocity of the mantle and the inner core is given by

$\frac{\left|R_{N} C_{N}^{10}\right|}{\left|R_{i c} C_{i c}^{10}\right|}=\frac{\sum_{j=1}^{i c}[\rho]_{j} R_{j}^{3}}{\sum_{j=i c+1}^{N}[\rho]_{j} R_{j}^{3}}$

For the different models of Mercury, the amplitude of the mantle displacement can reach nearly $15 \%$ of the amplitude of the inner core displacement, so that the motion of the mantle cannot be neglected (see Fig. 3). For the case of the Earth, the amplitude of the mantle displacement is smaller than $0.1 \%$ of that of the inner core (Grinfeld \& Wisdom 2005) because of the relatively small inner core of the Earth compared to these considered for Mercury. Different colors are used to represent different initial sulfur contents of the core. We will always use the same colors in our different figures.

\subsection{Newton's second law for the inner core}

The forces exerted on the inner core are the gravitational force and the pressure force applied at the ICB. Newton's second law for the inner core is therefore

$M_{I} \boldsymbol{a}=-\int_{V_{I}} \rho \nabla \psi \mathrm{d} \Omega-\int_{S_{I}} p N \mathrm{~d} S$,

where $N$ is the outward normal to the inner core boundary, $p$ the pressure applied on the surface of the inner core and $\psi$ the gravitational potential. We denote the total mass of the inner 


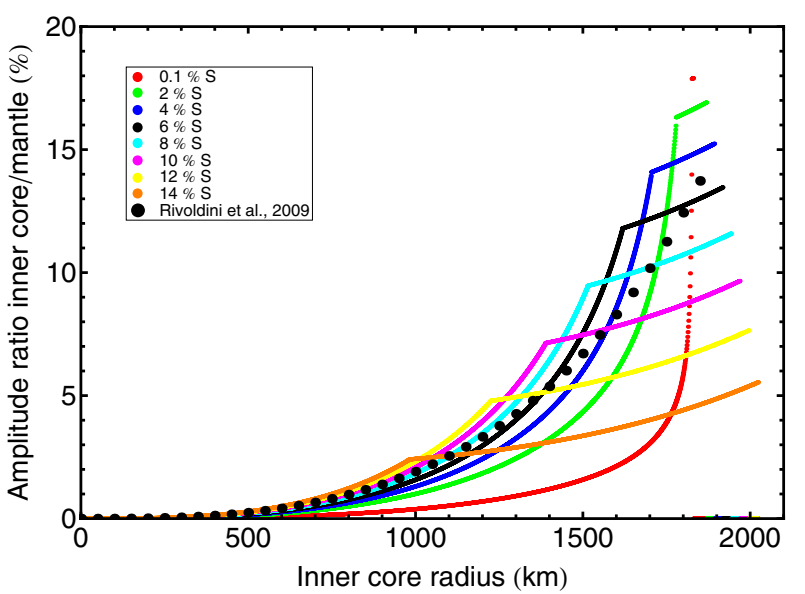

Fig. 3. Amplitude ratio of the mantle and the inner core velocity for the three and four-layer models of Mercury. Different colors represent different initial sulfur contents of the core, from $0.1 \mathrm{wt} \%$ to $14 \mathrm{wt} \%$. Amplitude ratio for full internal structure models of Mercury (Rivoldini et al. 2009) are represented with big bullets.

core by $M_{I}$, its volume by $V_{I}$ and its surface by $S_{I}$. In the volume $V_{I}$ of the inner core, we can distinguish ic spheres of volumes $V_{1}, \ldots, V_{i c}$, with radius $R_{1}, \ldots, R_{i c}$, density $[\rho]_{1}, \ldots,[\rho]_{i c-1}$ and $\rho_{i c}$. Newton's second law can then be expressed as

$$
M_{I} \boldsymbol{a}=-\sum_{j=1}^{i c-1} \int_{V_{j}}[\rho]_{j} \nabla \psi \mathrm{d} \Omega-\int_{V_{c}} \rho_{i c} \nabla \psi \mathrm{d} \Omega-\int_{S_{I}} p \boldsymbol{N} \mathrm{d} S,
$$

we take the time derivative of this equation and use the theorems of Reynolds and Green to transform the right-hand side of this equation. By also assuming that the outer core is inviscid $(\nabla \cdot v=0)$, it follows that Newton's second law can be written as

$$
\begin{aligned}
M_{I} \frac{\mathrm{d} \boldsymbol{a}}{\mathrm{d} t}= & -\sum_{j=1}^{i c-1} \int_{S_{j}}\left([\rho]_{j} \frac{\partial \psi}{\partial t} \boldsymbol{N}+C_{j}[\rho]_{j} \nabla \psi\right) \mathrm{d} S \\
& -\int_{S_{I}}\left(\rho_{i c} \frac{\partial \psi}{\partial t} \boldsymbol{N}+C_{i c} \rho_{i c} \nabla \psi+C_{i c} \nabla p+\frac{\partial p}{\partial t} \boldsymbol{N}\right) \mathrm{d} S .
\end{aligned}
$$

Since the amplitude of the Slichter modes is expected to be small, we linearize Newton's second law by writing the timedependent gravitational potential $\psi(t, r)$ and pressure $p(t)$ as $\psi(t, r)=\psi_{0}(r)+\bar{\psi}(t, r)$ and $p(t)=p_{0}+\bar{p}(t)$, where $\psi_{0}$ and $p_{0}$ are the gravitational potential and pressure at rest and $\bar{\psi}$ and $\bar{p}$ are small increments. In the outer core, $\psi_{0}$ and $p_{0}$ satisfy the equation for hydrostatic equilibrium, so that at the bottom of the outer core, we have

$\nabla p_{0}+\rho_{\mathrm{o}} \nabla \psi_{0}=0$

where $\rho_{\mathrm{o}}$ is the density of the outer core. By only keeping terms up to the first order in the interface velocities, we then obtain

$$
\begin{aligned}
M_{I} \frac{\mathrm{d} \boldsymbol{a}}{\mathrm{d} t}= & -\sum_{j=1}^{i c-1} \int_{S_{j}}\left([\rho]_{j} \frac{\partial \psi}{\partial t} \boldsymbol{N}+C_{j}[\rho]_{j} \nabla \psi_{0}\right) \mathrm{d} S \\
& -\int_{S_{I}}\left(\left(\rho_{i c} \frac{\partial \psi}{\partial t}+\frac{\partial p}{\partial t}\right) \boldsymbol{N}+C_{i c}\left(\rho_{i c}-\rho_{o}\right) \nabla \psi_{0}\right) \mathrm{d} S
\end{aligned}
$$

As we can restrict ourselves to an oscillation of the inner core along the $\hat{z}$ axis, we project this equation onto this axis, which gives

$$
\begin{aligned}
M_{I} \frac{\mathrm{d} a^{z}}{\mathrm{~d} t}= & -\sqrt{\frac{4 \pi}{3}}\left[\sum_{j=1}^{i c-1} \int_{S_{j}}[\rho]_{j}\left(\frac{\partial \psi}{\partial t}+C_{j} \frac{\partial \psi_{0}}{\partial r}\right) Y_{10} \mathrm{~d} S\right. \\
& \left.+\int_{S_{I}}\left(\left(\rho_{i c} \frac{\partial \psi}{\partial t}+\frac{\partial p}{\partial t}\right)+C_{i c}\left(\rho_{i c}-\rho_{o}\right) \frac{\partial \psi_{0}}{\partial r}\right) Y_{10} \mathrm{~d} S\right]
\end{aligned}
$$

where we have used $\boldsymbol{a} \cdot \hat{z}=a^{z}, \boldsymbol{N} \cdot \hat{z}=\cos \theta$ and $\nabla \psi_{0} \cdot \hat{z}=$ $\partial \psi_{0} / \partial r \cos \theta$. The factor $\sqrt{4 \pi / 3}$ appears due to the normalisation of the spherical harmonics $Y_{10}(\theta, \phi)=\sqrt{3 / 4 \pi} \cos \theta$. Below we develop the terms $\partial \psi_{0} / \partial r, \partial \psi / \partial t$ and $\partial p / \partial t$ in the inner core and at the inner core boundary.

The gravitational potential $\psi(r)$ inside and outside a spherical and homogeneous mass distribution of radius $R$ and density $\rho$ is given by

$$
\psi(r)=\frac{4 \pi}{3} G \rho \begin{cases}\frac{1}{2} r^{2}-\frac{3}{2} R^{2} & \text { if } \quad r \leqslant R \\ -R^{3} r^{-1} & \text { if } \quad r \geqslant R .\end{cases}
$$

Since we have $N$ mass distributions with radius $R_{j}$ and densities $[\rho]_{j}$, the gravitational potential $\psi_{0}$ in the $k$ th layer of the inner core $(k<i c)$ is

$\psi_{0}(r)=\frac{4 \pi}{3} G\left(\frac{1}{2} \rho_{k} r^{2}-\frac{3}{2} \sum_{j=k}^{N}[\rho]_{j} R_{j}^{2}-\sum_{j=1}^{k-1}[\rho]_{j} R_{j}^{3} r^{-1}\right)$

and we have

$\left.\frac{\partial \psi_{0}}{\partial r}\right|_{S_{k}}=\frac{4 \pi}{3} G\left(\rho_{k} R_{k}+\sum_{j=1}^{k-1}[\rho]_{j} R_{j}^{3} R_{k}^{-2}\right)$

As the gravitational potential verifies $\nabla^{2} \psi=4 \pi G \rho$, we see that $\partial \psi / \partial t$ is harmonic and can be written as

$\frac{\partial \psi_{j}}{\partial t}=\left(S_{j}^{l m}\left(\frac{r}{R_{j}}\right)^{l}+T_{j}^{l m}\left(\frac{r}{R_{j}}\right)^{-l-1}\right) Y_{l m}(\theta, \phi)$

in each spherical mass distribution $j$. The gravitational potential must be finite at the origin and must disappear at infinity. Therefore, inside the mass distribution, $T_{j}^{l m}$ must be equal to zero while outside the mass distribution, $S_{j}^{l m}=0$. Imposing continuity of $\partial \psi / \partial t$ at the surface $S_{j}$ leads to the equality of the $S$-coefficient inside the mass distribution to the $T$-coefficient outside the mass distribution. Since the jump in the normal spatial derivative of the time variation of the potential at the surface $S_{j}$ is equal to $4 \pi G C_{j}[\rho]_{j}$, the coefficient $S_{j}^{l m}$ is given by

$S_{j}^{l m}=-\frac{4 \pi}{2 l+1} G[\rho]_{j} R^{2} \mathrm{i} \omega \mathrm{e}^{\mathrm{i} \omega t} C^{l m} Y_{l m}$

and the time derivative of the gravitational potential $\psi_{j}$ of the spherically symmetric mass distribution with radius $R_{j}$ and mass density $[\rho]_{j}$ is

$\frac{\partial \psi_{j}}{\partial t}=-\frac{4 \pi G[\rho]_{j} R_{j}^{2} \mathrm{i} \omega \mathrm{e}^{\mathrm{i} \omega t} C_{j}^{l m}}{2 l+1} Y_{l m} \begin{cases}\left(\frac{r}{R_{j}}\right)^{l} & r \leqslant R_{j} \\ \left(\frac{r}{R_{j}}\right)^{-l-1} & r \geqslant R_{j} .\end{cases}$ 
Again, we have here $N$ mass distributions with radius $R_{j}$ and densities $[\rho]_{j}$. As we are interested in oscillations along the $\hat{z}$ axis, we can restrict ourself to harmonics $l=1$ and $m=0$. In the $k$ th layer of the inner core, $\partial \psi / \partial t$ is then given by

$$
\frac{\partial \psi}{\partial t}=-\frac{4 \pi}{3} G Y_{10} \mathrm{i} \omega \mathrm{e}^{\mathrm{i} \omega t}\left(\sum_{j=1}^{k-1}[\rho]_{j} R_{j}^{4} r^{-2} C_{j}^{10}+\sum_{j=k}^{N}[\rho]_{j} R_{j} r C_{j}^{10}\right)
$$

so that, since the normal velocities at the surface of the different layers of the inner core and mantle are equal,

$$
\begin{aligned}
&\left.\frac{\partial \psi}{\partial t}\right|_{S_{k}}=-\frac{4 \pi}{3} G Y_{10} \mathrm{i} \omega \mathrm{e}^{\mathrm{i} \omega t}\left[\rho_{o} R_{k} R_{N} C_{N}^{10}\right. \\
&\left.+\left(\sum_{j=1}^{k-1}[\rho]_{j} R_{j}^{3} R_{k}^{-2}+\sum_{j=k}^{i c}[\rho]_{j} R_{k}\right) R_{i c} C_{i c}^{10}\right] .
\end{aligned}
$$

In order to obtain an expression for $\partial p / \partial t$, the outer core is assumed to be incompressible, inviscid and homogeneous with density $\rho_{\mathrm{o}}$. Pressure and velocity are given by Euler's equation of motion and the incompressibility condition

$$
\begin{aligned}
\frac{\partial \boldsymbol{v}}{\partial t}+\boldsymbol{v} \cdot \nabla \boldsymbol{v} & =-\frac{1}{\rho_{\mathrm{o}}} \nabla p-\nabla \psi \\
\nabla \cdot \boldsymbol{v} & =0 .
\end{aligned}
$$

We linearize these equations by expressing $\boldsymbol{v}$ and $p$ around the equilibrium state $\left(\boldsymbol{v}_{0}=0\right.$ and $\left.p_{0}\right)$ as $\boldsymbol{v}(t ; r, \theta, \phi)=\overline{\boldsymbol{v}}(r, \theta, \phi)$ and $p(t ; r, \theta, \phi)=p_{0}(r, \theta, \phi)+\bar{p}(r, \theta, \phi)$, where $\overline{\boldsymbol{v}}$ and $\bar{p}$ are the velocity and pressure perturbation.

By taking the time derivative of Eq. (38) and neglecting quadratic terms in $\overline{\boldsymbol{v}}$, we obtain

$\frac{\partial^{2} \overline{\boldsymbol{v}}}{\partial t^{2}}=-\frac{1}{\rho_{\mathrm{o}}} \nabla \frac{\partial \bar{p}}{\partial t}-\nabla \frac{\partial \psi}{\partial t}$

By taking the divergence of this equation and using equation (39) and Poisson's equation, we see that also $\partial \bar{p} / \partial t$ is harmonic, and $\bar{p}(r, \theta, \phi)$ can therefore be written as

$\bar{p}(r, \theta, \phi)=\rho_{\mathrm{o}} \omega^{2} P^{10}(r) Y_{10}(\theta, \phi) \mathrm{e}^{\mathrm{i} \omega t}$

with $P^{10}(r)=P_{+}^{10} r+P_{-}^{10} r^{-2}$. In order to determine $P_{+}^{10}$ and $P_{-}^{10}$, we write, from $(35)$

$$
\begin{aligned}
\frac{\partial \psi}{\partial t} & =-\frac{4 \pi}{3} G Y_{10} \mathrm{i} \omega \mathrm{e}^{\mathrm{i} \omega t}\left(\sum_{j=1}^{i c}[\rho]_{j} R_{j}^{3} r^{-2} R_{i c} C_{i c}^{10}+\rho_{o} r R_{N} C_{N}^{10}\right) \\
& \equiv-\frac{4 \pi}{3} G Y_{10} \mathrm{i} \omega \mathrm{e}^{\mathrm{i} \omega t}\left(A^{10} r+B^{10} r^{-2}\right) .
\end{aligned}
$$

We define $H^{10}(r) \equiv-\left(A^{10} r+B^{10} r^{-2}\right)$. By substituting (41) and (42) into Eq. (40) we obtain expressions for $\overline{\boldsymbol{v}}^{r}, \overline{\boldsymbol{v}}^{\theta}$ and $\overline{\boldsymbol{v}}^{\phi}$ :

$$
\begin{aligned}
& \overline{\boldsymbol{v}}^{r}=\mathrm{i} \omega F^{10}(r) Y_{10}(\theta, \phi) \mathrm{e}^{\mathrm{i} \omega t} \\
& \overline{\boldsymbol{v}}^{\theta}=\mathrm{i} \omega r^{-1} G^{10}(r) \frac{\partial Y_{10}(\theta, \phi)}{\partial \theta} \mathrm{e}^{\mathrm{i} \omega t} \\
& \overline{\boldsymbol{v}}^{\phi}=0,
\end{aligned}
$$

with

$$
\begin{aligned}
& F^{10}(r)=\frac{\mathrm{d} P^{10}}{\mathrm{~d} r}+\frac{1}{\omega^{2}} \frac{4 \pi}{3} G \frac{\mathrm{d} H^{10}}{\mathrm{~d} r} \\
& G^{10}(r)=P^{10}(r)+\frac{1}{\omega^{2}} \frac{4 \pi}{3} G H^{10}
\end{aligned}
$$

By imposing continuity of the normal components of the velocities of the inner and outer cores at the ICB and of the velocities of the outer core and the mantle at the CMB, we have ${ }^{1}$

$\left(\begin{array}{l}R_{i c} C_{i c}^{10} \\ R_{N} C_{N}^{10}\end{array}\right)=\left(\begin{array}{cc}1 & -2 R_{i c}^{-3} \\ 1 & -2 R_{N}^{-3}\end{array}\right)\left(\begin{array}{l}P_{+}^{10}-\frac{1}{\omega^{2}} \frac{4 \pi}{3} G A^{10} \\ P_{-}^{10}-\frac{1}{\omega^{2}} \frac{4 \pi}{3} G B^{10}\end{array}\right)$.

Finally, the time derivative of Eq. (41) at the inner core surface is

$\left.\frac{\partial p}{\partial t}\right|_{S_{I}}=\mathrm{i} \omega \rho_{\mathrm{o}} \omega^{2}\left(P_{+}^{10} R_{i c}+P_{-}^{10} R_{i c}^{-2}\right) Y_{10}(\theta, \phi) \mathrm{e}^{\mathrm{i} \omega t}$

with $P_{+}^{10}$ and $P_{-}^{10}$ given by (46).

\subsection{Slichter mode frequency}

Since the time derivative of the $z$-component of the acceleration of the inner core can be expressed as

$\frac{\mathrm{d} a^{z}}{\mathrm{~d} t}=-\sqrt{\frac{3}{4 \pi}} \mathrm{i} \omega^{3} R_{i c} C_{i c}^{10} \mathrm{e}^{\mathrm{i} \omega t}$,

Newton's second law can be rewritten, by replacing $\partial \psi_{0} / \partial r$, $\partial \psi / \partial t$ and $\partial p / \partial t$ by their expressions (32), (37) and (47), as a linear algebraic equation in $C_{i c}^{10}$ and $C_{N}^{10}$. To find the Slichter mode frequency $\omega$, we then use the fact that a homogeneous linear system of equations (here the system composed of the equation of conservation of momentum (Eq. (22)) and the Newton's second law for the inner core) has a nonzero solution if and only if its determinant is equal to zero. For the models for the interior structure of Mercury developed in Sect. 2, the periods are on the order of a few hours and increase with increasing inner core radius to infinite period (no more oscillations) when the whole core is solid. The periods obtained show that the smaller the density jump at the ICB, the longer the periods. The jump in the Slichter mode period once the outer core reach the eutectic composition can then be explained by the fact that the density jump at the ICB is smaller. Moreover, the increase of the Slichter mode period with increasing inner core radius is due to the larger inertia of the inner core. The Slichter mode periods for a selected set of more general models of Mercury (Rivoldini et al. 2009) have also been calculated for comparison. Those models confirm the trends found for the models with a few homogeneous layers, with an increase of the Slichter mode period with increasing inner core radius (see Fig. 4).

For a three-layer model of Mercury, the Slichter frequency can be expressed as (Grinfeld \& Wisdom 2005)

$\omega^{2}=\frac{4 \pi}{3} G \rho_{2} \frac{D M}{\frac{3}{2} E M+D(M-m)}$

with $M$ the mass of Mercury, $m$ the difference between the mass of the inner core and the mass of a body with the volume of the inner core and density of the outer core, $D=\left(\rho_{1}-\rho_{2}\right)\left(R_{2}^{3}-R_{1}^{3}\right)$ and $E=\rho_{2} R_{2}^{3}$. Compared to the period for four-layer models of Mercury when the outer core is at the eutectic composition, the Slichter mode periods are a few hours to more than $100 \mathrm{~h}$ shorter, corresponding to a relative difference in period that ranges from 50 to almost $100 \%$ (Fig. 5).

\footnotetext{
${ }^{1}$ Note that in their article, Grinfeld \& Wisdom (2005) have omitted the factors $R_{i c}$ and $R_{N}$ before $C_{i c}^{10}$ and $C_{N}^{10}$.
} 
A. Coyette et al.: Period of the Slichter mode of Mercury and its possible observation

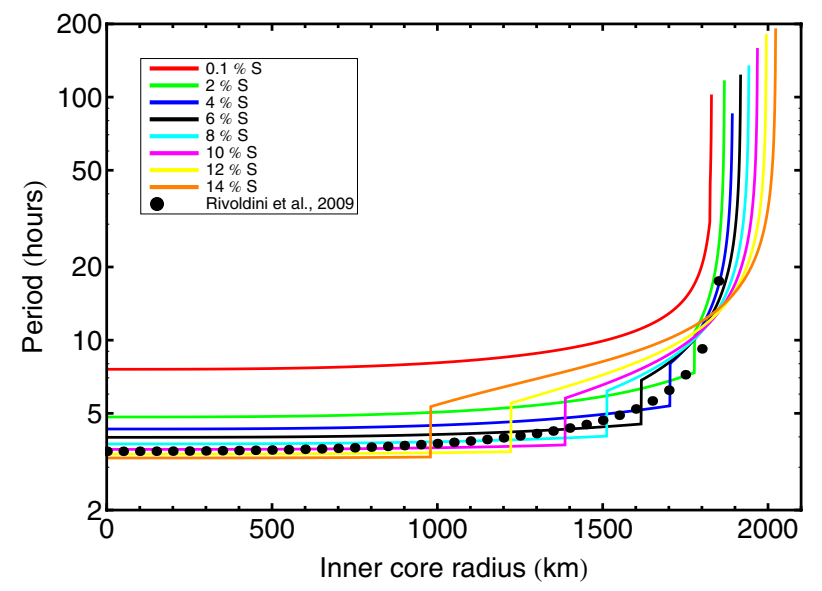

Fig. 4. Slichter mode periods for the different models of Mercury. Legend used is the same as in Fig. 3.

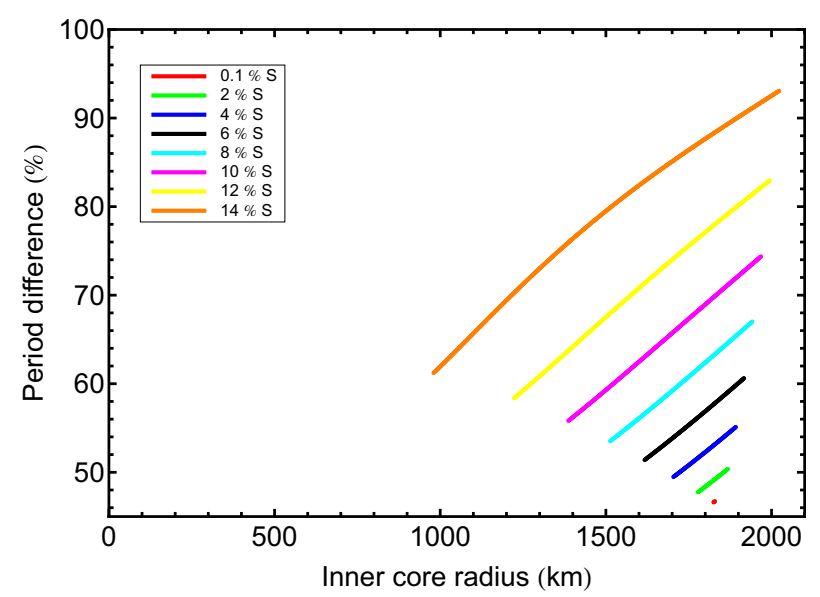

Fig. 5. Difference between the periods obtained for four-layer models of Mercury and for three-layer models of Mercury. Legend used is the same as in Fig. 3.

If the motion of the mantle (i.e. $C_{N}^{10}=0$ ) is neglected, the frequency can be expressed as (Busse 1974)

$\omega^{2}=\frac{4 \pi}{3} G \rho_{2} \frac{2 D}{3 E+2 D}$.

Although the motion of the mantle can be as large as $15 \%$ of the motion of the inner core, neglecting the mantle motion reduces the Slichter mode period by less than one minute for models with three layers (Fig. 6). This surprising result is probably due to the fact that the contribution of the mantle motion to the Slichter mode period is reduced by the low density difference between the inner and the outer cores (Escapa \& Fukushima 2011).

When the inner core is very small compared to the outer core, the frequency of the Slichter mode can be approximated as

$\omega^{2} \approx \frac{4 \pi}{3} G \rho_{2} \frac{2\left(\rho_{1}-\rho_{2}\right)}{2 \rho_{1}+\rho_{2}} \approx \frac{8 \pi G}{9}\left(\rho_{1}-\rho_{2}\right)$,

so that, when the inner core is small, the Slichter mode frequency depends mainly on the density jump at the ICB. This explains why the Slichter mode periods of Mercury are quite similar to that of the Earth when the inner core is small. It also explains the difference in periods with density jump at the ICB observed in Fig. 4.

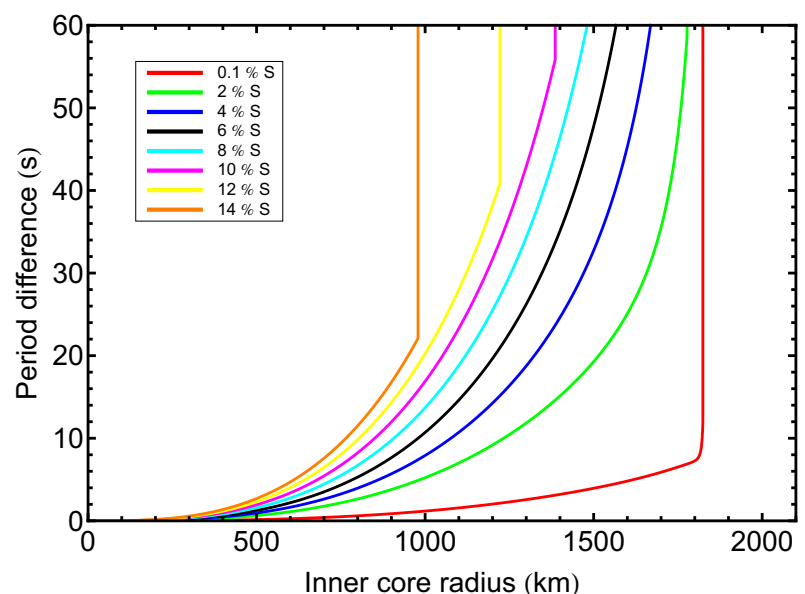

Fig. 6. Difference between Slichter mode periods obtained with and without mantle motion for the three layers models of Mercury. Legend used is the same as in Fig. 3.

\section{Slichter modes detection}

We assess here whether Slichter modes of Mercury can be detected by spacecraft. Probably the best way to detect Slichter modes of Mercury is to measure the surface gravity anomalies due to the motion of the inner core of Mercury with a lander (Grinfeld \& Wisdom 2005). If the inner core moves with an amplitude $A$, the mantle moves by conservation of momentum with an amplitude $B$

$B=\frac{\sum_{j=1}^{i c}[\rho]_{j} R_{j}^{3}}{\sum_{j=i c+1}^{N}[\rho]_{j} R_{j}^{3}} A$,

so that the inner core is a distance $\mathrm{A}+\mathrm{B}$ closer to the surface in the direction of motion of the inner core. In first order, the gravitational potential at a distance $r$ from the center of Mercury is given by

$\psi^{\prime} \approx-\frac{G M}{r}-\frac{G m}{r^{2}}(A+B)$.

The surface gravity anomaly is then

$\Delta \psi \approx \frac{G m}{r^{2}} \frac{M}{\sum_{j=i c+1}^{N}[\rho]_{j} R_{j}^{3}} A$.

As already stated by Grinfeld \& Wisdom (2005), the gravity anomaly due to the Slichter mode increases with increasing size of the inner core and can be up to two orders of magnitude greater than the expected gravity anomaly due to the Slichter mode of the Earth (less than 1 ngal, Hinderer et al. 2007) for a very large inner core.

As the center of mass of Mercury remains fixed during a Slichter mode oscillation, gravity anomalies due to this mode cannot be measured from orbit. However, the Slichter modes of Mercury can also be detected from orbit by measuring the motion of the mantle of Mercury. The precision of the surface position due to altimeter and orbit errors of MESSENGER and BepiColombo is respectively $10 \mathrm{~m}$ (Solomon et al. 2001) and smaller than $1 \mathrm{~m}$ (Iess et al. 2009). Figure 7 presents the amplitude of the inner core oscillations associated with a surface motion of $1 \mathrm{~m}$ (BepiColombo). For MESSENGER, the amplitudes are an order of magnitude larger. As expected, the smallest inner core displacement required for detection by MESSENGER or BepiColombo decreases with increasing inner core radius. 


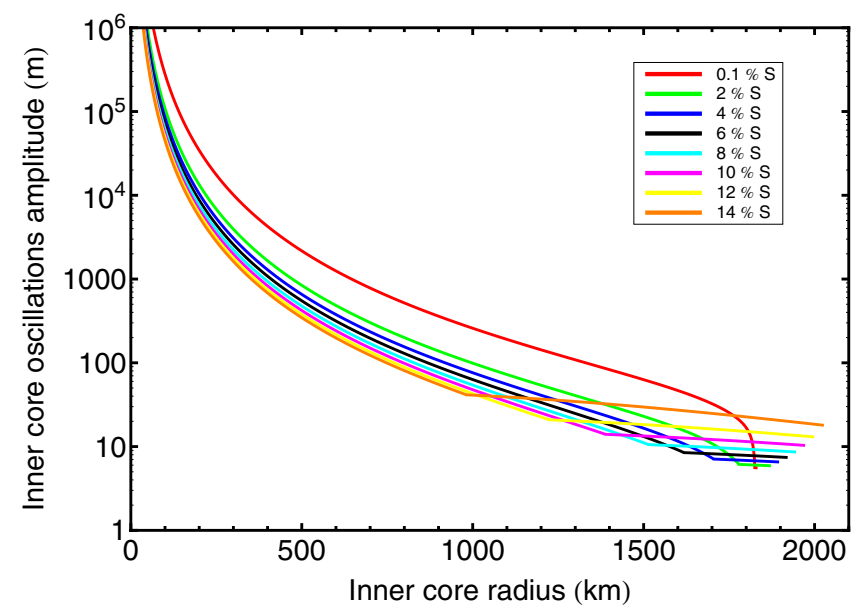

Fig. 7. Amplitude of the inner core oscillations that could lead to a detection of the Slichter mode by BepiColombo. Legend used is the same as in Fig. 3.

For inner core radii smaller than a few hundred $\mathrm{km}$, displacements on the order of one $\mathrm{km}$ or more are needed. For the largest inner cores, the displacement can be as small as ten meters and still allows detection.

We now assess whether a collision with a meteoroid can excite the Slichter modes to an observable level. In the collision with Mercury, most of the kinetic energy of the impacting body is converted into heat or spent in creating the impact crater. The energy efficiency $k$, which is the ratio of the seismic energy and the initial kinetic energy is given by (Shishkin 2007)

$k=2.8\left(\frac{\sigma}{S}\right)^{2}\left(\frac{R / R_{m}}{\alpha v_{m} / c_{p}}\right)^{2 / 3}$,

where $S$ is the shear modulus of Mercury, $\sigma$ its shear strength, $R$ its radius, $R_{m}$ the radius of the impacting body, $c_{p}$ is the propagation velocity of the longitudinal waves, $v_{m}$ the impact velocity and $\alpha=2 g R / c_{p}^{2}$ ( $g$ is the gravity at Mercury's surface). For Mercury, we will use $\sigma / \mu=2.4 \times 10^{-3}$ and $c_{p}=7.35 \mathrm{~km} \mathrm{~s}^{-1}$ (Shishkin 2007). As the gravity at the surface of Mercury is $g=3.70 \mathrm{~m} \mathrm{~s}^{-2}$, we obtain $\alpha=0.33$. For an impact velocity of $30 \mathrm{~km} \mathrm{~s}^{-1}$ (mean impact velocity, Killen et al. 2007), the energy efficiency of a meteoroid impact is then on the order of $10^{-2}$ for a meteoroid with a radius of some hundred meters and $5 \times 10^{-4}$ for a radius of $10 \mathrm{~km}$.

Although all the seismic modes are excited by a collision (Smith 1976; Rosat \& Rogister 2012), we consider that all the energy remaining is transfered to the Slichter mode of Mercury as an ideal favorable case. Therefore, we have

$k \frac{m_{m} v_{m}^{2}}{2}=\frac{M_{m} v^{2}}{2}+\frac{M_{I} V^{2}}{2}$

where $m_{m}$ is the mass of the impact body, $M_{m}$ and $v^{\prime}$ is the mass and the velocity of the mantle and $M_{I}$ and $V$ the mass and the velocity of the inner core of Mercury. Considering that the magnitude of the oscillation velocity of the inner core and mantle can be expressed as $v^{\prime}=\omega B$ and $V=\omega A=\omega \eta B$ (where $\eta$ is given by $(52)$ ), the mass of the meteoroid needed to excite the Slichter mode to as surface displacement amplitude $B$ is expressed as

$m_{m}=\frac{\omega^{2} B^{2}\left(M_{m}+M_{I} \eta^{2}\right)}{k v_{m}^{2}}$.

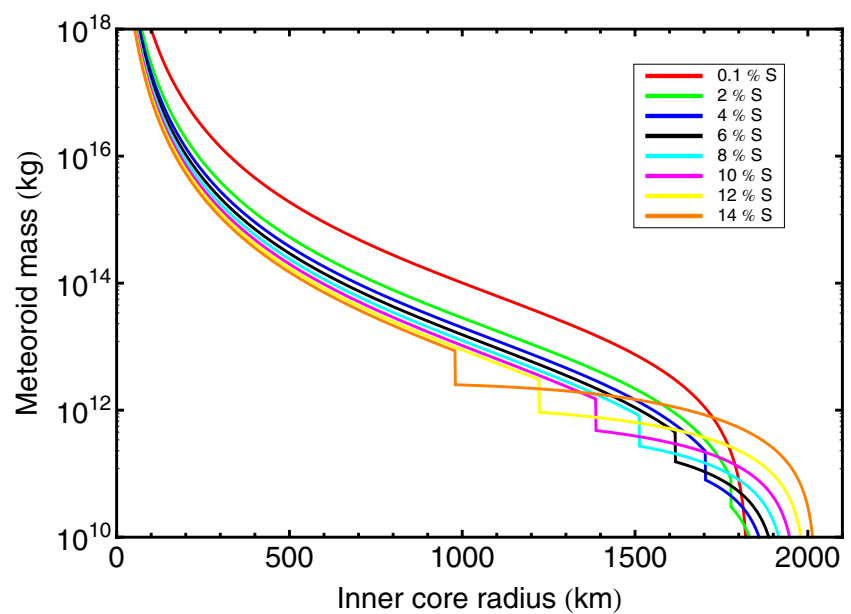

Fig. 8. Mass of the meteoroid that could lead to a detection of the Slichter mode by BepiColombo assuming that all the remaining energy is transfered to the Slichter mode. Legend used is the same as in Fig. 3.

However, $k$ varies with the radius of the meteoroid and therefore with its mass. This dependence can be written as

$k=2.8\left(\frac{\sigma}{S}\right)^{2}\left(\frac{R}{\alpha v_{m} / c_{p}}\right)^{2 / 3}\left(\frac{4 \pi \rho_{m}}{3}\right)^{2 / 9} m_{m}^{-2 / 9} \equiv \tilde{k} m_{m}^{-2 / 9}$

assuming that the meteoroid is spherical with a mean density $\rho_{m}=1000 \mathrm{~kg} / \mathrm{m}^{3}$. The mass of the meteoroid needed to excite the Slichter mode to a surface displacement amplitude $B$ is then

$m_{m}=\left(\frac{\omega^{2} B^{2}\left(M_{m}+M_{I} \eta^{2}\right)}{\tilde{k} v_{m}^{2}}\right)^{9 / 7}$.

The Slichter mode could be detected by BepiColombo $(B=1 \mathrm{~m})$ if the mass of the meteoroid is at least on the order of $10^{16} \mathrm{~kg}$ (meteoroid of about $10 \mathrm{~km}$ radius) for a small inner core but the minimal mass decreases up to about $10^{10} \mathrm{~kg}$ (meteoroid of about $100 \mathrm{~m}$ radius) for a large inner core (Fig. 8). As the rate of impact of Jupiter family comets of radius greater than $1 \mathrm{~km}$ on Mercury is about $8.6 \times 10^{-10}$ meteoroids per year (Levison \& Duncan 1997), the Slichter mode excitation of Mercury would occur in average one time over a billion years if the inner core is very small. However, the average interval between two impacts is $140 \mathrm{My}$ for a $100 \mathrm{~m}$ radius meteoroid (Marchi et al. 2005) so that, when the inner core is large, the Slichter mode of Mercury could be more frequently excited.

Holsapple (1993) has developed scaling laws describing the properties of a crater impact from the radius and velocity of the impactor. When the gravity dominates the strength of the materials in the formation of the crater, the rim radius of a simple bowl-shaped crater is given by (Holsapple 1993)

$R_{\mathrm{sc}}=14.95 g^{-0.17} R_{m}^{0.83} v_{m}^{0.34}$

where the units of $g, R_{m}$ and $v_{m}$ are respectively $\mathrm{m} \mathrm{s}^{-2}$, $\mathrm{m}$ and $\mathrm{km} \mathrm{s}^{-1}$. For an impactor of $10 \mathrm{~km}$ radius, the rim radius is of the order of $80 \mathrm{~km}$. However, when the crater radius is greater than a transition value of $R_{*}=5 \mathrm{~km}$ (Pike 1988), the slumping of the walls leads to a complex crater of radius

$R_{c c}=\left(1.02 \frac{R_{\mathrm{sc}}}{R_{*}^{0.079}}\right)^{1.086}$.

Using this last formula, an impact of a meteoroid or comet of $10 \mathrm{~km}$ radius and a velocity of $30 \mathrm{~km} \mathrm{~s}^{-1}$ would leave a crater 
Table 2. Magnetic properties of Mercury.

\begin{tabular}{lccc}
\hline \hline Magnetic properties & Notations & Values & References \\
\hline Radial magnetic field & $B_{r}$ & $0.01 \mathrm{mT}$ & 1 \\
strength in the core & & & \\
Electrical conductivity & $\sigma$ & $6 \times 10^{5} \mathrm{~S} \mathrm{~m}^{-1}$ & 2 \\
Magnetic permeability & $\mu$ & $4 \times 10^{-7} \pi \mathrm{H} \mathrm{m}^{-1}$ & 2 \\
Magnetic diffusivity & $\eta$ & $1.33 \mathrm{~m}^{2} \mathrm{~s}^{-1}$ & 2 \\
\hline
\end{tabular}

References. (1) Dumberry (2011); (2) Wicht et al. (2007).

of more than $100 \mathrm{~km}$ radius on the surface of Mercury. For an impact velocity of $30 \mathrm{~km} \mathrm{~s}^{-1}$, the rim radius is as small as $1.7 \mathrm{~km}$ for an impactor with a radius of $100 \mathrm{~m}$. A near-global catalog of craters with a radius greater than $10 \mathrm{~km}$ has recently been compiled from Mariner 10 and MESSENGER images (Fassett et al. 2011). It remains to be studied whether a suitable impact exists.

Until now, we have considered that once excited the oscillations of the inner core of Mercury continue forever. The introduction of dissipative processes leads to a damping of the Slichter mode. If we neglect the deformation of the inner core during Slichter mode oscillations, the damping of the Slichter mode is principaly due to magnetic and viscous stresses in the outer core (Buffett \& Goertz 1995; Rieutord 2002). As the viscosity in the outer core of Mercury is still unknown, we consider here only dissipation due to a magnetic field.

The correction to the Slichter mode frequency due to magnetic stresses is given by (Buffett \& Goertz 1995)

$$
\frac{\delta \omega}{\omega}=\frac{3}{4} \Lambda \frac{L}{R_{i c}} \frac{\rho_{o c}}{\rho_{i c}} \frac{(1+i) \sqrt{1-i \Lambda}}{1-i \Lambda+\sqrt{1-i \Lambda}}
$$

where $\Lambda \approx B_{r}^{2} /\left(L^{2} \omega^{2} \mu \rho_{o c}\right)$ is the ratio of the magnetic force to the inertial force and $L=\sqrt{2 \eta / \omega}$ is the depth of the magnetic boundary layer at the ICB (skin depth). Subscript $i c$ is used for the inner core and subscript $o c$ for the outer core. The different magnetic properties of Mercury (radial magnetic field strength in the core $B_{r}$, electrical conductivity $\sigma$, magnetic permeability $\mu$ and magnetic diffusivity $\eta$ ) used here are given in Table 2.

The e-folding time $\tau$ is given by $\tau=[\mathfrak{J}(\delta \omega)]^{-1}$. For a small inner core, the damping time is about $10 \mathrm{ky}$ (see Fig. 9), so that the observation of the Slichter mode should be very unlikely with an excitation one time over a billion years. When the inner core is very large, the damping time is about $0.5 \mathrm{My}$, still below the average time between impacts of $100 \mathrm{~m}$ radius meteoroid. Moreover, as we have considered here only dissipation due to the magnetic field of Mercury, the effective damping time will be smaller than $0.5 \mathrm{My}$. The observation of the Slichter mode of Mercury, if excited by meteoroid impacts, would therefore require a fortunate recent impact.

\section{Discussion and conclusion}

In order to study the Slichter modes of Mercury, we have developed models of the interior structure of Mercury consisting of three and four homogeneous layers. We have generalized the methodology developed by Grinfeld \& Wisdom (2005) for the study of the Slichter modes to a planet with more than three layers. We have taken into account the motion of the planet's mantle, even if, as stated by Grinfeld \& Wisdom (2005), the Slichter mode periods are only little influenced by this motion. Nevertheless, this motion is needed to study the possibility of observing the Slichter mode, as this observation could be performed by measuring the motion of the mantle. The Slichter

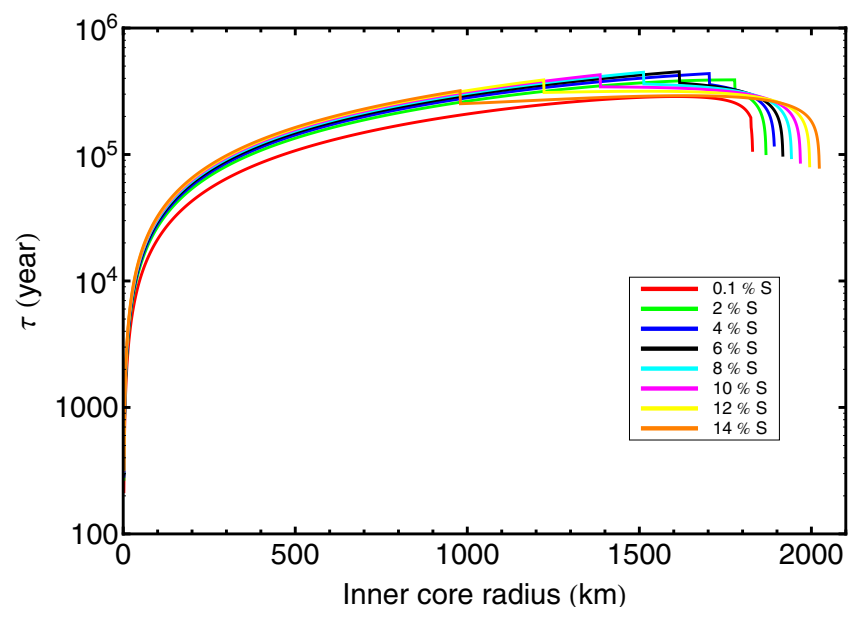

Fig. 9. Magnetic damping time of the Slichter mode of Mercury. Legend used is the same as in Fig. 3.

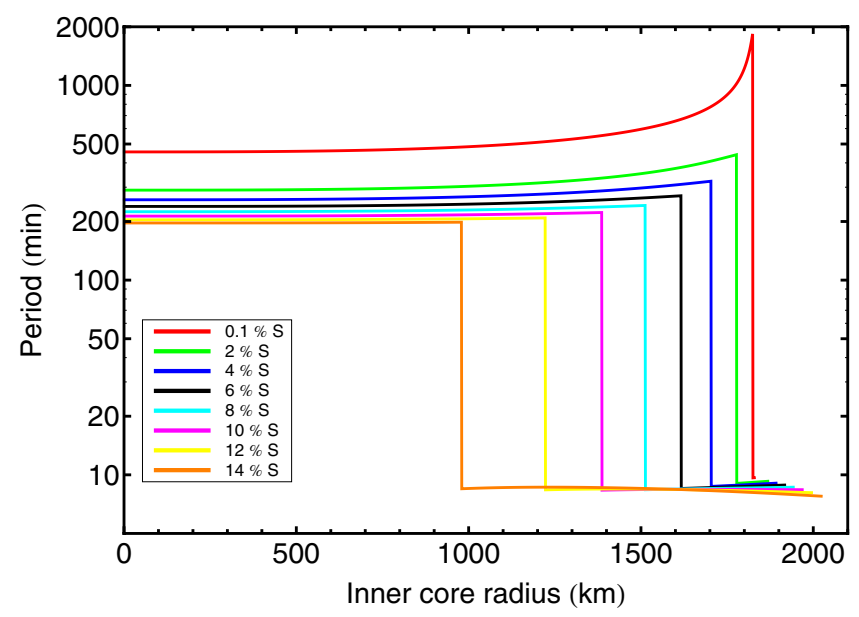

Fig. 10. Slichter mode periods taking into account instantaneous phase transformations at the ICB when the outer core reaches the eutectic composition. Legend used is the same as in Fig. 3.

mode period obtained ranges from a few hours to more than $100 \mathrm{~h}$ and depends strongly on the properties of Mercury's core. If the period is on the order of a few hours, it mainly depends on the density jump at the inner core boundary and observation of the period would constrain the composition of the core. If Mercury has a Slichter mode period longer than ten hours, the radius of the inner core would be at least $1700 \mathrm{~km}$.

When the outer core reaches the eutectic composition, new solid deposited on the inner core upon further cooling has the same composition as the liquid and the composition of the outer layers of the inner core at the ICB is similar to that of the outer core. Phase transformation at the ICB can then be associated with oscillations of the inner core. Following the methodology of Grinfeld \& Wisdom (2010) generalized to a four-layer planet, we calculated the period of the Slichter mode by taking into account instantaneous phase transformations. The Slichter mode periods are shortened by an order of magnitude and are on the order of ten minutes (see Fig. 10). However, as already stated above, it seems unrealistic that the outer core of Mercury has already reached the eutectic composition.

Measuring gravity anomalies due to the Slichter mode cannot be performed from orbit as the center of mass of Mercury remains fixed during the Slichter mode oscillations. We have therefore studied the possible observation of Slichter modes of 
Mercury by the probes MESSENGER and BepiColombo by measuring the motion of the mantle of Mercury using an altimeter. We have shown that the observation of the Slichter mode of Mercury is possible if the inner core is very large and is excited to an amplitude of the order or greater than ten meters. If the Slichter mode of Mercury is assumed to be excited by a collision with a meteoroid, the mass of the meteoroid must be at least of the order of $10^{10} \mathrm{~kg}$ (for a large inner core). On average, such a collision occurs once every $100 \mathrm{My}$, which is much larger than the dissipation time of the Slichter mode, therefore requiring a recent impact (less than $0.5 \mathrm{My}$ ago).

Acknowledgements. We thank the anonymous referee for useful comments and suggestions.

\section{References}

Aharonson, O., Zuber, M. T., \& Solomon, S. C. 2004, Earth Planet. Sci. Lett., 218,261

Ahrens, T. J., Holland, K. G., \& Chen, G. Q. 2002, Geophys. Res. Lett., 29, 54 Anderson, W. W., \& Ahrens, T. J. 1994, J. Geophys. Res., 99, 4273

Anderson, B. J., Acuña, M. H., Korth, H., et al. 2008, Science, 321, 82

Breuer, D., Hauck II, S. A., Buske, M., Pauer, M., \& Spohn, T. 2007, Space Sci. Rev., 132, 229

Buffett, B. A., \& Goertz, D. E. 1995, Geophys. J. Int., 120, 103

Busse, F. H. 1974, J. Geophys. Res., 79, 753

Courtier, N., Ducarme, B., Goodkind, J., et al. 2000, Phys. Earth Planet. Int., 117,3

Dreibus, G., \& Wänke, H. 1985, Meteoritics, 20, 367

Dumberry, M. 2011, Geophys. Res. Lett., 38, L16202

Escapa, A., \& Fukushima, T. 2011, AJ, 141, 77

Fassett, C. I., Kadish, S. J., Head, J. W., Solomon, S. C., \& Strom, R. G. 2011, Geophys. Res. Lett., 38, L10202

Giampieri, G., \& Balogh, A. 2002, Planet. Space Sci., 50, 757

Grinfeld, P., \& Wisdom, J. 2005, Phys. Earth Planet. Int., 151, 77

Grinfeld, P., \& Wisdom, J. 2010, Phys. Earth Planet. Int., 178, 183

Hauck II, S. A., Dombard, A. J., Phillips, R. J., \& Solomon, S. C. 2004, Earth Planet. Sci. Let., 222, 713

Hinderer, J., Crossley, D., \& Jensen, O. 1995, Phys. Earth Planet. Int., 90, 183

Hinderer, J., Crossley, D., \& Warburton, R. 2007, in Treatise on Geophysics, ed.

T. Herring, \& G. Schubert (Amsterdam: Elsevier), 3, 65

Holsapple, K. A. 1993, Annu. Rev. Earth Planet. Sci., 21, 333
Iess, L., Asmar, S., \& Tortora, P. 2009, Acta Astronautica, 65, 666

Jensen, O., Hinderer, J., \& Crossley, D. J. 1995, Phys. Earth Planet. Int., 90, 169

Kaiura, G. H., \& Toguri, J. M. 1979, Can. Mettal. Quart., 18, 155

Killen, R., Cremonese, G., Lammer, H., et al. 2007, Space Sci. Rev., 132, 433

Kroner, C., Jahr, T., \& Jentzsch, G. 2004, J. Geodyn., 38, 263

Levison, H. F., \& Duncan, M. J. 1997, Icarus, 127, 13

Li, J., \& Fei, Y. 2005, in Treatise on Geochemistry: The Mantle and Core, ed. R. Carlson (New-York: Elsevier), 2, 521

Li, J., Fei, Y., Mao, H. K., Hirose, K., \& Shieh, S. R. 2001, Earth Planet. Sci. Let., 193, 509

Marchi, S., Morbidelli, A., \& Cremonese, G. 2005, A\&A, 431, 1123

Margot, J. L., Peale, S. J., Jurgens, R. F., Slade, M. A., \& Holin, I. V. 2007, Science, 316, 710

Melosh, H., \& McKinnon, W. 1988, in Mercury, ed. F. Vilas, C. R. Chapman, \& M. Matthews (Tucson: The University of Arizona Press), 374

Nishida, K., Suzuki, A., Ohtani, E., et al. 2008, Equation of state for liquid FeS at high pressure and temperature

Oberst, J., Elgner, S., Turner, F. S., et al. 2011, Planet. Space Sci.

Ono, S., Oganov, A. R., Brodholt, J. P., et al. 2008, Earth Planet. Sci. Lett., 272, 481

Perry, M. E., Kahan, D. S., Barnouin, O. S., et al. 2011, Planet. Space Sci.

Pike, R. J. 1988, in Mercury, ed. F. Vilas, C. R. Chapman, \& M. S. Matthews (Tucson: The University of Arizona Press), 165

Rieutord, M. 2002, Phys. Earth Planet. Int., 131, 269

Rivoldini, A., Van Hoolst, T., \& Verhoeven, O. 2009, Icarus, 201, 12

Rivoldini, A., Van Hoolst, T., Verhoeven, O., Mocquet, A., \& Dehant, V. 2011, Icarus, 213, 451

Rosat, S., \& Rogister, Y. 2012, Phys. Earth Planet. Int., 190, 25

Rosat, S., Sailhac, P., \& Gegout, P. 2007, Geophys. J. Int., 171, 55

Shishkin, N. I. 2007, J. Appl. Mech. Tech. Phys., 48, 145

Slichter, L. B. 1961, Proc. Natl. Acad. Sci. USA, 47, 186

Smith, M. L. 1976, J. Geophys. Res., 81, 3055

Smylie, D. E. 1992, Science, 255, 1678

Solomon, S. C., McNutt Jr., R. L., Gold, R. E., et al. 2001, Planet. Space Sci., 49,1445

Sun, H., Zheng, D., Ding, X., Chen, W., \& Chen, X. 2006, Chin. Sci. Bull., 51, 713

Urakawa, S., Someya, K., Terasaki, H., et al. 2004, Phys. Earth Planet. Int., 143, 469

Van Hoolst, T., \& Jacobs, C. 2003, J. Geophys. Res., 108, 5121

Watters, T. R., Robinson, M. S., Bina, C. R., \& Spudis, P. D. 2004, Geophys. Res. Lett., 31, L04701

Watters, T. R., Solomon, S. C., Robinson, M. S., et al. 2009, Earth Planet. Sci. Lett., 285, 283

Wicht, J., Mandea, M., Takahashi, F., et al. 2007, Space Sci. Rev., 132, 261

Xu, J., Sun, H., \& Zhou, J. 2010, Chin. Sci. Bull., 55, 276 